\title{
Report of the IAU WGAS Sub-group on Numerical Standards
}

\author{
E. M. Standish, Chair \\ $J P L /$ Caltech \\ s01-150; Pasadena, CA 91109; USA \\ ems@smyles.jpl.nasa.gov
}

\begin{abstract}
.
The Report of the Sub-Group on Numerical Standards of the IAU Working Group on Astronomical Standards (WGAS) is presented. The report is intended to incorporate the majority of the responses received from the e-mail recipients of the series of WGAS Circulars. The report proposes to retain the present (1976) IAU System of Astronomical Constants and also proposes to establish a IAU File of Current Best Estimates. Further, the report proposes the establishment of a Maintenance Committee, governed by a set of proposed by-laws, in order to oversee the maintenance of the new file.
\end{abstract}

\section{Introduction}

The present LAU System of Astronomical Constants provides a stable standard for the consistent reduction of observations. However, such a system is inadequate for current high-precision astronomy. Thus, it is desirable to both retain the present system and to also provide a more current set of values. Furthermore, since improved techniques of measurement now require not only more accurate numerical constants, but also more sophisticated formulation, consideration must be given to coordinating the presentation of the numerical constants with associated algorithms and formulations.

Therefore, it is proposed to

1. Retain the present "1976 IAU System of Astronomical Constants".

2. Establish an "IAU File of Current Best Estimates" whereby the accuracy of current astronomical data computations is made available to the astronomical community.

3. Establish a "Maintenance Committee" to oversee the maintenance of the File of Current Best Estimates.

4. Establish a set of By-Laws to govern the actions of the Maintenance Committee.

This paper presents an initial File of Current Best Estimates, an initial set of guidelines for the establishment of a Maintenance Committee to govern the file, and an initial set of By-Laws to govern the Maintenance Committee. The initial File of Current Best Estimates is discussed in the next section and the file itself is given in the Table I; the By-Laws and the establishment of the Maintenance Committee are given in Sections 3 and 4.

\section{The 1994 IAU File of Current Best Estimates}

Table I presents the 1994 IAU File of Current Best Estimates.

When the difference is significant, values are given for both the previously used TDB units and for the newly adopted SI units. The value of $L_{G}$ comes directly from $W_{O} / c^{2}$ For the conversion of $\tau_{A}$ and $c \tau_{A}$ into SI units, the TDB values are multiplied by the factor $\left(1+L_{B}\right)^{(1 / 3)}$; for $G_{E}$ and $G_{S}$, the TDB values are multiplied by the factor $\left(1+L_{B}\right)$.

The uncertainties, given in parentheses, are intended to be: realistic values, as opposed to formal ones.

For cases where there are two or more valid source: for the same constant, each is given.

180

1. Appenzeller (ed.), Highlights of Astronomy, Vol. 10, 180-184.

(1995 IAU. Printed in the Netherlands. 


\section{By-Laws Governing the IAU File of Current Best Estimates}

This section presents an initial set of by-laws to govern the IAU File of Current Best Estimates. The contents and maintenance of the File are outlined first; the composition of the Maintenance Committee and amendments to the By-Laws are discussed afterward.

\subsection{AUTHORITY}

There is to be a Maintenance Committee whose purpose is to maintain the IAU File of Current Best Estimates. The Committee is to be governed by this present set of By-Laws.

\subsection{Contents of the File of Current Best Estimates}

The IAU File of Current Best Estimates is to contain a list of selected astronomical parameters. For each parameter, the list is to include

1. an alphameric name of no more than 6 characters,

2. the current best estimate of its numerical value,

3. an estimate of its realistic uncertainty (standard deviation),

4. a description of the physical units in which the quantity is expressed,

5. a reference to the source of the value,

6. an identifying name or definition of the parameter.

\subsection{SCOPE OF THE FILE}

The File is to include any parameter which is contained in the IAU System of Astronomical Constants and for which there is a more accurately determined value. In addition, the File may contain values for other descriptive parameters of astronomical objects. Objects to be considered in the future include the planets, satellites, asteroids, comets, sun, and extra-solar-system objects; descriptive parameters of these objects may be masses, shapes, gravity fields, orientation parameters, etc. Consideration should also be given to ephemerides, catalogues, station locations, plate motions, etc.

When deciding which parameters are to be included, care is to be taken not to usurp the jurisdiction of other scientific groups or to create inconsistencies by providing alternate values of quantities which are given elsewhere.

\subsection{Selection of Parameter Values}

The Maintenance Committee is to maintain quality control over the values to be included in the File. The Committee is to consider values of parameters provided by any legitimate source, not necessarily restricted to published values. The Committee may also decide to automatically adopt certain Files from other IAU Commissions or from other scientific sources. However, the Maintenance Committee is to be responsible for the integrity of the File; it must decide whether a parameter is appropriate for inclusion in the File and whether a suggested value is reliable. Furthermore, the Committee must decide whether a published value should be altered (for purposes of round-off, correction of errors, change of units, etc.). The Committee must decide which units are most appropriate for each parameter.

\subsection{Updating The File: Frequency and Schedule}

The IAU File of Current Best Estimates is to be updated only at the beginning of a calendar year and only if it is deemed, by the Maintenance Committee, that there is a significant reason for doing so. 
In order for the value of a parameter to be changed or to be included in the File, the Maintenance Committee must receive, electronically or in writing, the proposed action on or before the first of September. The Maintenance Cornmittee is to formally accept or reject any proposed addition or change on or before the first of December. The newly amended File is to be sent to the Distribution Centers by December 15; the Distribution Centers are to incorporate the updated File during the last half of December.

\subsection{ARChiving}

The name of each File is to include the calendar year during which the File was the active IAU File of Current Best Estimates. The active File and all previously active Files are to be immediately accessible, electronically, from the designated Distribution Centers. All Files are also to be permanently archived on suitable electronic media, such as CDrom's, magnetic tapes, etc.

\subsection{Distribution}

There is to be a central Distribution Center, chosen by the Maintenance Committee, which will, in turn, distribute the two files to a number of secondary distribution centers, located so as to provide comfortable access around the world. The files are to be made available in hard copy as well as via some suitable electronic medium such as anonymous FTP, electronic-mail, or electronic bulletin boards. The distribution process may be coordinated with the Sub-Group on Standard Procedures.

\subsection{AMENDMENT OF The By-LaWs}

Subsequent amendments to these By-Laws are to be presented to and voted upon by all members of the IAU Commissions represented in the enabling Joint Commission (IAU Commissions 4, 5, 8, 19, 24, and 31). Proposed amendments must be presented four weeks in advance of the voting; acceptance is by a two-thirds majority of all of the voting members.

\subsection{Composition of the Maintenance Commititee}

The composition of the Maintenance Committee is to reflect the range of disciplines represented by the sponsoring Joint Commission (IAU Commissions 4, 5, 8, 19, 24, 31). The composition should also display a wide national and geographic distribution.

The Committee is to include, but not be limited to, a Chair, a Vice Chair, and liaison members from the IERS standards committee, the IUGG standards committee, the IAU Cartographic Coordinates Working Group and each of the sponsoring IAU Commissions.

The members and officers of the Maintenance Committee are to assume their duties at the conclusion of each IAU General Assembly. The terms of Chair and Vice-Chair are three years each; the Vice- Chair normally assumes the role of Chair at the time of change. The term for a normal Committee member is six years; these should be staggered so that approximately half of the members are replaced at each time of change. The lengths of the terms of the liaison members may be left unspecified.

\section{Initial Maintenance Committee for the IAU File of Current Best Estimates}

The initial Maintenance Committee is to be appointed by the current Chair of the IAU Working Group on Astronomical Standards along with the other three leaders of the WGAS Sub-Groups. The selection process is to follow the guidelines set forth in Section 3.9 of the By-Laws. The acceptance of the initial Maintenance Committee is to require a majority vote of all members of IAU Commissions $4,5,8,19,24$, and 31 . 


\section{Conclusions}

The mechanism of a two-tiered approach to supplying astronomical constants has been proposed. The presently used standard system of astronomical constants is to be retained and is to be accessible electronically. A second file is to be established which contains state-of-the-art values. Further, an archival system is proposed whereby any past set of values can be uniquely retrieved. Maintenance of the files is given by a set of by-laws.

\section{Acknowledgements}

The research in this paper was carried out by the Jet Propulsion Laboratory, California Institute of Technology, under a contract with the National Aeronautics and Space Administration.

\section{References}

1. Anderson,J.D., Colombo,G., Esposito,P.B., Lau,E.L. and Trager,G.B.: 1987, "The Mass, Gravity Field, and Ephemeris of Mercury", Icarus, 71, 337-349.

2. Sjogren,W.L., Trager,G.B. and Roldan,G.R.: 1990, "Venus: A Total Mass Estimate", Geophys. Res. Let., 17(10), $1485-1488$.

3. JPL Planetary and Lunar Ephemeris DE245

4. Null,G.W.: 1969, "A Solution for the Mass and Dynamical Oblateness of Mars Using Mariner-IV Doppler Data", Bull. Am. Astr. Soc., 1(4), 356.

5. Campbell,J.K. and Synnott,S.P.: 1985, "Gravity Field of the Jovian System from Pioneer and Voyager Tracking Data", Astron. J., 90(2), 364-372.

6. Campbell,J.K. and Anderson,J.D.: 1989, "Gravity Field of the Saturnian System from Pioneer and Voyager Tracking Data ${ }^{n}$, Astron. J., 97(5), 1485-1495.

7. Jacobson,R.A., Campbell,J.K., Taylor,A.H. and Synnott,S.P.: 1992, “The Masses of Uranus and its Major Satellites from Voyager Tracking Data and Earth-based Uranian Satellite Data", Astron J., 103(6), 2068-2078.

8. Jacobson,R.A., Riedel,J.E. and Taylor,A.H.: 1991, "The Orbits of Triton and Nereid from Spacecraft. and Earthbased Observations", Astron. Astrophys., 247, 565-575.

9. Tholen,D.J, and Buie,M.W.: 1988, "Circumstances for Pluto-Charon Mutual Events in 1989", Astron.J., 96(6), 1977-1982.

10. Fukushima,T.: 1994, "Time Ephemeris", to be published in Astron. Astrophys.

11. Bursa,M., Sima,Z., Kostelecky, J.: 1992, "Determination of the-Geopotential Scale Factor from Satellite Altimetry", Studia geoph. et geod., 36, 101-114.

12. Ries,J.C., Eanes, R.J., Shum,C.K. and Watkins,M.M.: 1992, "Progress in the Determination of the Ciravitational Coefficient of the Earth", GRL, 19(6), 529-531.

13. Williams,J.G., Newhall, $X X$ and Dickey,J.O.: 1991, "Luni-Solar Precession: Determination from Lunar Laser Ranges”, Astron. Astrophys., 241, L9-L12.

14. Miyamoto,M. \& Soma,M.: 1993, "Is the Vorticity Vector of the Galaxy Perpendicular to the Galactic Plane? I. Precessional Correction and Equinoctial Motion Correction to the FK5 System", Astron J., 105, 691.

15. Bursa,M.: 1992, "Parameters of Common Relevance of Astronomy, Geodesy and Geodynamics", Bull. Geod., 66(2), 193-197.

16. Moritz,H.: 1992, “Geodetic Reference System", Bull. Geod., 66(2), 187-192.

17. Rapp: 1994, private communication via E. Groten. 
TABLE I

1994 IAU File of Current Best Estimates

\begin{tabular}{|c|c|c|c|c|c|}
\hline Constant & Alphameric & Value & Units & Ref & Description \\
\hline \multicolumn{6}{|c|}{ Deflning Constants } \\
\hline$k$ & GAUSSK & 0.01720209895 & {$\left[a u^{3} / d a y^{2}\right]^{1 / 2}$} & & Gaussian gravitational constant \\
\hline$c$ & CLIGHT & 299792458 & $\mathrm{~m} / \mathrm{s}$ & & Speed of light \\
\hline \multicolumn{6}{|c|}{ Primary Constants } \\
\hline$L_{C}$ & LSUBC & $1.4808268452(1) \times 10^{-8}$ & & {$[10]$} & Mean of $d(T C B) / d(T C G)-1$ \\
\hline \multirow[t]{2}{*}{$\tau_{A}$} & TAUA & $499.00478642(7)$ & $S I$ is & {$[3]$} & Astronomical unit \\
\hline & & $499.00478384(7)$ & $T D B: s$ & & \\
\hline$p$ & PREC & $5028.83(4)$ & $" / c t y$ & {$[13,14]$} & General precession in longitude \\
\hline$\epsilon$ & OBLIQ & $84381.412(5)$ & $n$ & [3] & Obliquity of ecliptic $\mathrm{J} 2000$ \\
\hline $\mathcal{M}_{\text {Sun }} / \mathcal{M}_{1}$ & MRAT1 & $6023600 .(250)$. & & [1] & Mass Ratio: Sun/Mercury \\
\hline $\mathcal{M}_{\text {Sun }} / \mathcal{M}_{2}$ & MRAT2 & $408523.71(6)$ & & [2] & Mass Ratio: Sun/Venus \\
\hline$M_{S u n} / M_{B}$ & MRATB & $328900.56(2)$ & & [3] & Mass Ratio: Sun/(Earth+Moon) \\
\hline $\mathcal{M}_{\text {sun }} / \mathcal{M}_{4}$ & MRAT4 & 3098708.(9.) & & [4] & Mass Ratio: Sun/Mars system \\
\hline $\mathcal{M}_{\text {sun }} / \mathcal{M}_{s}$ & MRAT5 & $1047.3486(8)$ & & [5] & Mass Ratio: Sun/Jupiter system \\
\hline $\mathcal{M}_{\text {sun }} / \mathcal{M}_{6}$ & MRAT6 & $3497.898(18)$ & & [6] & Mass Ratio: Sun/Saturn system \\
\hline $\mathcal{M}_{\text {sun }} / \mathcal{M}_{7}$ & MRAT7 & $22902.98(3)$ & & {$[7]$} & Mass Ratio: Sun/Uranus system \\
\hline $\mathcal{M}_{\text {Sun }} / \mathcal{M}_{s}$ & MRAT8 & $19412.24(4)$ & & [8] & Mass Ratio: Sun/Neptune system \\
\hline $\mathcal{M}_{S_{\mathrm{un}}} / \mathcal{M}_{\mathrm{q}}$ & MRAT9 & $1.35(7) \times 10^{8}$ & & [9] & Mass Ratio: Sun/Pluto system \\
\hline $\mathcal{M}_{\text {Moon }} / \mathcal{M}_{\text {Earth }}$ & MERAT & $0.012300034(3)$ & & [3] & Mass Ratio: Moon/Earth \\
\hline$G$ & GRAV & $6.67259(30) \times 10^{-8}$ & $m^{3} /\left(g s^{2}\right)$ & {$[15,16]$} & Constant of Gravitation \\
\hline \multirow[t]{2}{*}{$G_{E}$} & GME & $398600.4415(8) \times 10^{9}$ & $S I: \mathrm{m}^{3} / \mathrm{s}^{2}$ & [12] & Geocentric gravitational constant \\
\hline & & $398600.4356(8) \times 10^{9}$ & $T D B: \mathrm{m}^{3} / \mathrm{s}^{2}$ & & \\
\hline$a_{\Sigma}$ & $\mathbf{R E}$ & $6378136.55(1)$ & $m-$ & {$[17]$} & $\begin{array}{l}\text { Mean equatorial radius of Earth's } \\
\text { ellipsoid }\end{array}$ \\
\hline \multirow[t]{2}{*}{$W_{0}$} & wo & $62636857.5(1.0)$ & $m^{2} / s^{2}$ & [11] & Potential of the geoid \\
\hline & & $62636856.26(1.0)$ & & {$[17]$} & \\
\hline$J_{2}$ & $\mathrm{~J} 2 \mathrm{E}$ & $1082.6269(6) \times 10^{-6}$ & & {$[15,16]$} & $\begin{array}{l}\text { Zonal 2nd-degree geopotential } \\
\text { parameter }\end{array}$ \\
\hline $1 / f$ & FLATTG & $1 / 298.257(1)$ & & {$[15,16]$} & Flattening factor for the Earth \\
\hline$\omega$ & ROTE & $7292115 . \times 10^{-1 i}$ & $\mathrm{rad} / \mathrm{s}$ & {$[15,16]$} & $\begin{array}{l}\text { Mean angular velocity of Earth's } \\
\text { rotation }\end{array}$ \\
\hline \multicolumn{6}{|c|}{ Derived Constants } \\
\hline$L_{B}$ & LSUBB & $1.550519747(3) \times 10^{-8}$ & & & Mean of $d(T C B) / d(T T)-1$ \\
\hline$L_{G}$ & LSUBG & $6.9692903(1) \times 10^{-10}$ & & & Mean of $d(T T) / d(T C G)-1$ \\
\hline \multirow[t]{2}{*}{ ctA } & CTAUA & 149597871475.(30.) & $S I: m$ & & Astronomical Unit \\
\hline & & $149597870700 .(30)$. & $T D B: m$ & & \\
\hline $\mathcal{M}_{\text {Earth }} / \mathcal{M}_{\text {Moon }}$ & EMRAT & $81.30059(1)$ & & & Mass Ratio: Earth/Moon \\
\hline \multirow[t]{2}{*}{$G_{s}$} & GMSUN & $1.327124 \times 10^{20}$ & $S I: m^{3} / s^{2}$ & & Heliocentric gravitational constant \\
\hline & & $1.32712442007 \times 10^{20}$ & $T D B: \mathrm{m}^{3} / \mathrm{s}^{2}$ & & \\
\hline $\mathcal{M}_{\text {Sun }} / \mathcal{M}_{\text {Earth }}$ & SERAT & $332946.05(2)$ & & & Mass Ratio: Sun/(Earth) \\
\hline
\end{tabular}

